\title{
The Effect of Intervention Program to Reduce Stress in Women Employees
}

\author{
Dr.ArtiTiwari*, Dr.SunitaBalani** \\ *Lecturer, Amity Institute OfBehavioral and Allied Sciences, Amity University, Lucknow \\ **Lecturer, Amity Institute OfBehavioral and Allied Sciences, Amity University, Lucknow
}

\begin{abstract}
The aim of the present study is to see the impact of intervention program on stress ofwomen employees. Stress can have a serious effect on the work output of women employees and on their physical and mental health. The data were collected from women employees of a private university of Lucknowusing the Perceived Stress Scale and a stress check list. After this an intervention programme was organised which includedstress inoculation training and hardiness training. The results revealed that after the intervention programmes there was significant change in the amount of stress among woman and it was confirmed by the post-test by using the same scale.Theresults also revealed that out of the list of 11 possible stressors, women employees in this sample identified an average of 6.3aspects of their job $(S D=2.7)$ as being stressful. The working hours, workload, lack of resources to carry out their job, and the low level ofreward were most frequently identified as stressors.

Keywords: stress, women employees, intervention programmes, SIT and Hardiness training, mental Health
\end{abstract}

\section{Introduction}

Women in India have come a long way after independence. From just a skilled homemaker, women today have acquired skills and capabilities of not just being a homemaker but being at par with their male counterparts. Stress has permeated our lives. We have learnt to cope with it. But there are times when stress overwhelms our lives, more so for working women who are walking the tight rope of family and professional commitments.(Anitha, 2007).

The modern urban woman wants to be a multi-taskeran efficient career woman, a loving mother, a caring wife and an active socialite. In this process, she gets stressed without even realizing it. In India, over half of the call centre employees feel so stressed out by the tough working conditions that they end up quitting. Stress is an adaptive response to a situation that is perceived as challenging or threatening to a person's well being. Stress is a negative consequence of modern living. People are stressed because of overwork, job insecurity, information overload and the increasing pace of life.

Hans Selye first introduced the concept of Stress into the life science in 1936. He defined stress as, "the force, pressure, or strain exerted up to a material object or person which resists these forces and attempttomaintainitsoriginalstate."

The phenomenon of stress has become very common in the modern complex world. In the fast changing world of rapid technological advancement, more and more people are becoming victims of stress and this is true of people in all organization and at all levels.

A study conducted by W.H.O. in 2001 reveals that every fourth person in the world is suffering from high level of stress, and by 2020, depression will be the world's second largest cause for urban disease after heart ailments(Bureau of Labour Statistics, 2001). The role of stress in everyday life therefore needs hardly to be over emphasized. Stress as well as its prevention and management has now become an integral and essential part of the modern life. The working female who still takes on the burden of responsibility at home and for the children may suffer from inevitable stress associated with trying to be "superwomen" (Nicholson, 1995).

Stress management is defined as any attempt to reduce the negative effects of stress. Management techniques can be divided into those which are broadly biological in nature and those which are broadly psychological in nature. Psychological methods of stress management try to control the body's response to stress by altering the way we think about the stressor. These methods include Stress Inoculation Training (SIT) and Hardiness Training.

\section{Stress Inoculation Training (SIT)}

SIT was developed by Meichenbaum (1976), who believes it should be undertaken before stress reaches a critical level. It is an example of a cognitive-behavioural approach to stress management. Its aim is to change the way we think about stress (cognitive restructuring). If we can change the way we think about stress, we can change the way we behave towards it. SIT assumes that people sometimes find situations stressful 
because they think about them in 'catastrophising' ways (i.e. they always think the worst). SIT tries to change negative ways of thinking to positive ways in three stages: (1) Cognitive preparation ('Conceptualisation'), (2) Skills training and practice (3) Real-life application ('Application and follow through')

Hardiness trainingpeople who score high on Kobasa's 3 Cs are less affected by stress. Presumably, stress could be reduced if people could be made hardier. Kobasa proposes three ways of increasing hardiness: (1) Focussing: Some people cannot recognise the physical signs of stress (e.g. tenseness). Since you can't deal with a stressor if you can't identify when you're stressed, Kobasa's first approach is to teach a person to recognise the physiological changes associated with stress. (2) Reliving stressful encounters: Even if a person can identify stress, the way they deal with it might not be appropriate. So, the persons are asked to think about a recent stressful situation, and how they could have dealt with it more effectively. (3) Self-improvement: This final stage involves learning to avoid stress in the future by thinking about and appraising potentially stressful situations differently. If a stressor cannot be avoided, or otherwise dealt with, the person is encouraged to take on a challenge which can be dealt with. This allows us to experience the positive aspects of coping with a stressor. The consequence of this is that we can 'bounce back' more readily from failure, and potentially serious stressors are experienced as being less stressful Present Study

Literature review suggests that women employees are subject to various stressors in their job and this could have a consequence on their physical and mental health. The objective of this study therefore was to explore the stressful aspects in women faculties of a private universityand to identify how faculties cope with stress. The specific questions of the study were: (1) Are the working women stressed? (2)Which aspects of the respondents' job were considered to be stressful? (3) How did stress inoculation training and hardiness training help the respondents to cope with stress? (4) Effects of intervention programmes for stress reduction?

\section{Participants}

\section{Method}

The sample comprised of 50 faculties working at a private university of different departments. The age of the respondents ranged from 30-45 years and they had served the institution for a period ranging from 10 months to8 years. The faculty were from department of hotel management, pharmacy, law, engineering technology, architecture and fashion technology. There were 44 married women employees in the sample and 06 unmarried women.

\section{Instruments}

A check list of eleven factors was used to identify aspects of the women employees' job that the respondents find stressful. These included work load, lack of resources to carry out the job, rewards, work hours, opportunity for advancement,and relationship with co-workers, time to spend on self, promotion and increments, keeping scheduling, relationship with seniors, personal enhancement.

The Perceived Stress Scale (PSS) by Cohen, Kamarck, and Mermelstein (1983) is used which is one of the few instruments to measure a global level of perceived stress, dealing with the degree to which situations in one's life are appraised as stressful. It is a14-item scale that assesses perceived stressful experience or stress responses over the previous month. Each item is on a five-point Likert format which requires scoring of $4=$ never, $3=$ almost never, $2=$ sometimes, $1=$ fairly often and $0=$ very often for items stating positive experiences or response. Reverse scoring is required for items stating negative experiences or response. Total possible scores are from 0 to 56. Higher scores represent high stress levels. There is an abundant literature re-porting re liability and validity of Perceived Stress Scale. The creators of the scale reported convergent validity indicated by relationships with depressive $(r=0.76 ; n=332)$ and physical $(r=0.70 ; n=64$ symptomatology scales. Internal consistency reliability was high with Cronbach's alpha coefficient ranging from0.84 $(n=332)$ to $0.86(n=64)$ (Cohen et al., 1983).

The PSS has subsequently been used in a range of settings and has been shown to relate to a number of physiological and psychological responses. Scores on the PSS are associated with depressive symptoms (Chang, 1998), measures of depression (Band Edelmann, Avery, \&Brinsden, 1998) and anxiety and psychosomatic complaints (van Eck, \& Nicolson, 1994). Intervention programmes for stress reduction was organised for five sessions which included stress inoculation training and hardiness training.

\section{Procedure}

Women facultiesfrom variousdepartments of a private university were contacted and the aims and objectives of the study were briefed. The data were collected through the use of a self-administered questionnaire and a checklist, which also had open-ended questions. Questionnaires and check lists were later sent out to all the departments and completed questionnaires were collected back on an agreed upon date. After receiving the 
data intervention programme was organised for five days which was of two hours daily to reduce their stress level.

Data analysis.

Statistical analysis was carried out using SPSS version 11.5 for windows. Percentages of responses were calculated for stressors for both the pre-test and post-test.

\section{Results}

The results revealed that the most stressful aspects for the faculties were working hours, resources to carry out the jobs,time to spend on self, keeping to schedule, promotion and increments. The mean stress score on PSS was 25.9 ( $\mathrm{SD}=6.01)$. A higher PSS score is indicative of higher stress levels. Out of the list of 11 possible stressors female respondents in this sample identified an average of $6.3(\mathrm{SD}=2.7)$ as being stressful aspects of their job. Lack of resources to carry out their job, the work-load, the low level of reward and the long working hours were the most frequently identified as stressors. Aspects of the job identified to be stressful are shown in Table 1.Interestingly, working relationships were relatively rarely identified as stressors suggesting that colleagues may be animportant source of support.

Table 1

\begin{tabular}{|c|c|}
\hline Aspects of job stress & Percentage \\
\hline Work hours & 92.4 \\
Workload & 90.2 \\
Expectation to be rewarded & 87.8 \\
Promotion and increment & 70.4 \\
Time to spend on self & 69.3 \\
Lack of resources to carry out the job & 63.2 \\
Keeping scheduling & 60.3 \\
Relationship with co-workers & 49.0 \\
Personal enhancement & 42.1 \\
Work related responsibilities & 36.3 \\
Relationship with seniors & 28.2 \\
\hline
\end{tabular}

Table 2.

Mean scores of pre and post PSS

\begin{tabular}{|l|c|c|c|c|l|}
\hline & Pre test & Post test & SD & t & $\underline{p}$ \\
\hline Mean & 40.5 & 28.7 & 5.67 & 14.56 & $<.01$ \\
\hline
\end{tabular}

\section{Discussion}

The objective of the study was to explore the stressful aspects in female respondents' jobs and the effects of intervention programme for stress reduction to cope with stress. Stress in female respondents was from the demanding nature of their work. If demands continue to rise and adjustments are not made, then inevitably a 'correction' will occur, which may take the form of 'burnout' or physical and/or mental impairment(Winefield, 2000)

The study identified female respondents' do face a considerable amount of stress at workplace. Woking hours and workload were identified to be the powerful source of stress. Because female respondents would have been expected to give more time to family and children. Expectation to get reward, promotion and increments were also identified to be a major source of stress.Blix(1999),Wiley (2005) and Javis (2002) also asserted that teachers experience significant amount of stress in the course of performing their duties. Because of so much involvement in job and family obligations female respondents get very less time to spend on themselves which is also a major source of stress.Freudenberger(1992)investigated those women who have to combine a professional role with that of family, experience more stress. Female respondents also expressed that they wanted to do something for their personal enhancement and to keep scheduling which also had been a source of stress in them.It is interesting to note that working relationships were relatively rarely identified as stress in our sample, therefore suggesting that colleagues may be an important source of support. In our sample, the mean stress score in the pre test was 40.5 and with a standard deviation of 5.67.This is considerably higher than the mean scores of post test which has been found 28.7. It shows that intervention programmes had a positive effect to reduce the stress level of the respondents. SIT had a positive effect upon the thinking of the respondents to change their negative ways of thinking into positive ways. In cognitive preparation or conceptualisation the respondents were trained to identify self defeating internal dialogue in potentially stressful situation and they 
were encouraged to think about why these negative self-statements occur. Next the respondents were given some skill training and practice to reduce stress for e.g. relaxation technique and more specific methods relating to themselves and their own stressors. These included 'preparation statements' which helped them to (1) prepare for a stressful situation. (2) Confront and handle the stressful situations and cope with the fear of being overwhelmed.(3)Finally the respondents were trained to face more threatening situations in which initially they were placed in a situation that was moderately easy to cope with. Once it has been mastered, a more difficult situation is presented progressively. A final strength of SIT is that it works. Research indicates that SIT is effective in reducing the stress associated with examinations and at least some phobias, and that in general it is a useful method for dealing with moderately stressful situations. In hardiness training respondents were trained in three ways to increase hardiness. In focussing stage, respondents were trained to recognise the physical signs of stress. In the second stage of reliving stressful encounters, respondents were practiced to face and deal with a stressful situation more effectively. In the final stage respondents were practiced to avoid stress in the future by thinking about and appraising potentially stressful situations differently. Like SIT, hardiness training is a problem-focused approach to stress management; these kinds of approaches are generally much more effective than problem-focussed approaches. Therefore, hardiness training would also appear to be a better way of managing stress.

Our study had a limitation of a small sample from the University. More studies on stress experienced by working female respondents India are essential to address this issue and to improve the quality of life working females for whom family is a priority.

\section{Conclusion}

The study identified that female respondents do face considerable amount of stress at workplace. Work hours and work load were identified to be the most stressful aspects of the job. It was therefore important to understand the nature of their work to get an insight into the origin of their stress and ways to prevent it. For this purpose intervention programme was organised with an aim to improve, balance and discover sustainable ways of remaining healthy while honouring the demands of the altruistic traditions of the profession. All the female respondents should be aware of predictors of risk and signals of impairment, as well as available avenues for assistance. A teacher's job should be experienced as inherently satisfying in response to a meaningful job well done. The prospect of a life time of joyless striving is unacceptable.It was found that the job outside home entails lot of dual responsibility to a woman. Still plenty remains to be seen through to keep a woman happy and satisfied.

\section{References}

[1]. Blix, A.G. (1999). Occupational stress among teachers.Educational Research 42(3), 162-170.

[2]. Bureau of LaborStatistics(2001). [Table R67] Number and percent distribution of nonfatal occupational injuries and illnesses involving days away from work by nature of injury or illness and number of days away from work, 2001.

[3]. Carlson, J.G., McGuigan, F. and SheppardJ.L. (Eds.), Stress and Health. Amsterdam: Harwood Academic (pp. 37-62).

[4]. Cohen, S., Kamarck, T., \&Mermelstein, R. (1983).A global measure of perceived stress. Journal of Health and Social Behavior,24, 385-396.

[5]. Cohen S.,\&Williamson G. (1988). Perceived stress in a probability sample of the United States. In: Spacapan S, Oskamps, editors.

[6]. Devi, A. S., 2007, Occupational stress: A comparative study of women indifferent occupations. Prajnan, 35(1), 61-7

[7]. Freudenberger,H.J.(1992), Understanding the caregiver. Psychotherapy in private practice,11,75-79 http:/www.bls.gov/iif/oshwc/osh/case/ostb1222.pdf

[8]. Jarvis, M. (2002).Teacher Stress. A Critical Review of recent Findings and Suggestions for future Research Direction. Stress News, $14(1), 52-56$

[9]. Kobasa,S.C.(1979): Stressful life events,Personslity and health: An inquiry into hardiness. Journal of personslity and social psychology,37,1-11

[10]. Meichenbaum, D. (1994):A clinical handbook/practical therapist manual for assessing and treating adults with post traumatic stress disorder. Waterloo, Ontario: Institute Press.

[11]. Meichenbaum, D., \&Deffenbacher, J. L. (1988). Stress inoculation training. Counseling Psychologist, 16, 69-90.

[12]. Nicholson, P. (1995).Gender power and organisation.London .Routledge.

[13]. Winefield, A. H. (2000). Stress in academe: somerecent research findings. In D.T. Kenny, Winefield, A. H., \& Jarrett, R. J. (2001).Occupational stress in university staff. InternationalJournal of Stress Management, 8, $285-298$.

[14]. Wiley, C. (2005) A synthesis of Research on the Causes, Effects, and Reduction Strategies of Teacher Stress, Journal of Instruction Psychology. 27, 1-9 